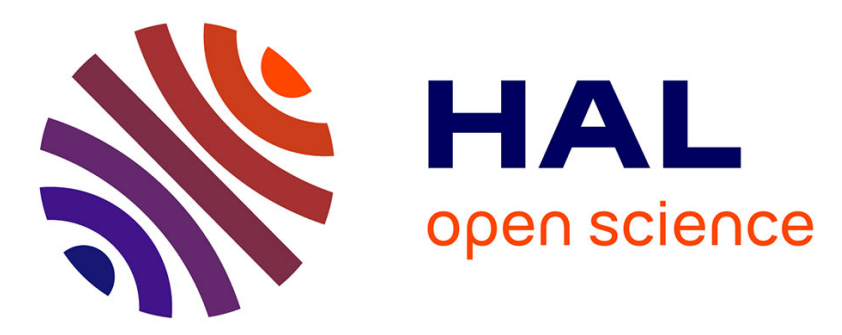

\title{
Global low-frequency oscillations in a separating boundary-layer flow
}

Uwe Ehrenstein, Francois Gallaire

\section{To cite this version:}

Uwe Ehrenstein, Francois Gallaire. Global low-frequency oscillations in a separating boundary-layer flow. IUTAM Symposium "Unsteady Separated Flows and their Control", Jun 2007, Corfu, Greece. pp.123-133. hal-00213445

\section{HAL Id: hal-00213445 \\ https://hal.science/hal-00213445}

Submitted on 23 Jan 2008

HAL is a multi-disciplinary open access archive for the deposit and dissemination of scientific research documents, whether they are published or not. The documents may come from teaching and research institutions in France or abroad, or from public or private research centers.
L'archive ouverte pluridisciplinaire HAL, est destinée au dépôt et à la diffusion de documents scientifiques de niveau recherche, publiés ou non, émanant des établissements d'enseignement et de recherche français ou étrangers, des laboratoires publics ou privés. 


\title{
Global low-frequency oscillations in a separating boundary- layer flow
}

\author{
Uwe Ehrenstein $^{1}$ and François Gallaire ${ }^{2}$ \\ ${ }^{1}$ IRPHÉ, Université de Provence, 49, Rue Joliot-Curie, F-13384 Marseille Cedex 13, France \\ ${ }^{2}$ Lab. J. A. Dieudonné, Université de Nice-Sophia Antipolis, Parc Valrose, F-06108 Nice Cedex \\ 02, France
}

\begin{abstract}
A separated boundary layer flow at the rear of a bump is considered and twodimensional flow states at increasing Reynolds numbers are computed using a nonlinear continuation procedure for the stationary Navier-Stokes system. The global instability analysis of the steady states is performed by computing two-dimensional temporal modes. The analysis reveals non-normal modes which are able to describe localized initial perturbations associated with large transient energy growth. At larger time a global low-frequency oscillation is found accompanied with periodic regeneration of the flow perturbation inside the bubble, as the consequence of nonnormal cancellation of modes. The initial condition provided by the optimal perturbation analysis is applied to Navier-Stokes time integration and is shown to trigger nonlinear 'flapping' typical for separation bubbles.
\end{abstract}

Key words: Boundary-layer separation, global instability, non-normality.

\section{Introduction}

There is general evidence that laminar detached boundary layers are likely to undergo two-dimensional low-frequency global oscillations [6] which have been observed both in experiments, whether the separation bubble is triggered by leading-edge geometries ([4]) or by adverse pressure gradients ([8], [11]). The physical mechanisms at the origin of this type of instability which occurs above a critical Reynolds number are only partially understood. For instance, the frequencies associated with possible transition from convective to absolute instability of local velocity profiles with a certain amount of reverse flow appear to be higher than the typical frequencies of the oscillations known as 'flapping' (cf. [9], [13]). By providing some evidence for the appearance of secondary recirculating flow regions at the rear of the main bubble, it has been conjectured ([5], [16]), that topological flow changes might be responsible for the overall instability behaviour. This possibility has been explored in [10] for a separated flow induced by a bump mounted on a flat plate but no clear-cut confirmation of topological flow changes could be given, in the absence of a basic state above criticality.

In this work we readdress the low-frequency oscillations for an elongated separation bubble induced by the bump geometry which has already been considered in [10]. We focus on the two-dimensional global instability behaviour. The three-dimensional 
transverse instability characterized by a global steady and weakly growing eigenmode has recently been analyzed in [7]. The resulting longitudinal instability first takes place when increasing the Reynolds number, which has also been observed in backward-facing step flows ([3], [2]). However, the low-frequency fluctuations which lead to an overall motion of the separation bubble is the dominant instability mechanism at higher Reynolds numbers.

The analysis combines a quasi-Newton approach to determine steady states for Reynolds numbers above criticality and a numerical method suitable for computing two-dimensional temporal modes. Section 2 is devoted to the description of the numerical tools. The global stability results are discussed in Section 3 and an optimal perturbation analysis is performed in Section 4. Optimal initial conditions are then used in the direct numerical simulation procedure and are shown to produce low-frequency 'flapping' described in Section 5. Some conclusions are provided in Section 6 .

\section{Numerical tools}

The flow domain is $0 \leq x \leq L, \eta(x) \leq y \leq H$, with $\eta(x)$ the lower boundary containing the bump which has already been considered in [10] (cf. figure 1 for the geometry). The height $H$ has been chosen large enough in order to recover uniform flow, the Navier-Stokes system being made dimensionless using the displacement thickness $\delta^{*}$ at inflow, where the Blasius profile is described, and the uniform freestream flow velocity $U_{\infty}^{*}$. The dimensionless bump height is $h=2$ and the mapping

$$
\bar{x}=\frac{2}{L} x-1, \quad \bar{y}=\frac{1}{H}(1-\gamma) y+\gamma \quad \text { with } \quad \gamma=\frac{H+\eta}{\eta-H}
$$

transforms the domain into $[-1,1] \times[-1,1]$. A Chebyshev-Chebyshev collocation discretization is used for the transformed system, together with a stretching in the wall-normal direction which redistributes the collocation points in order to take into account the boundary-layer structure.
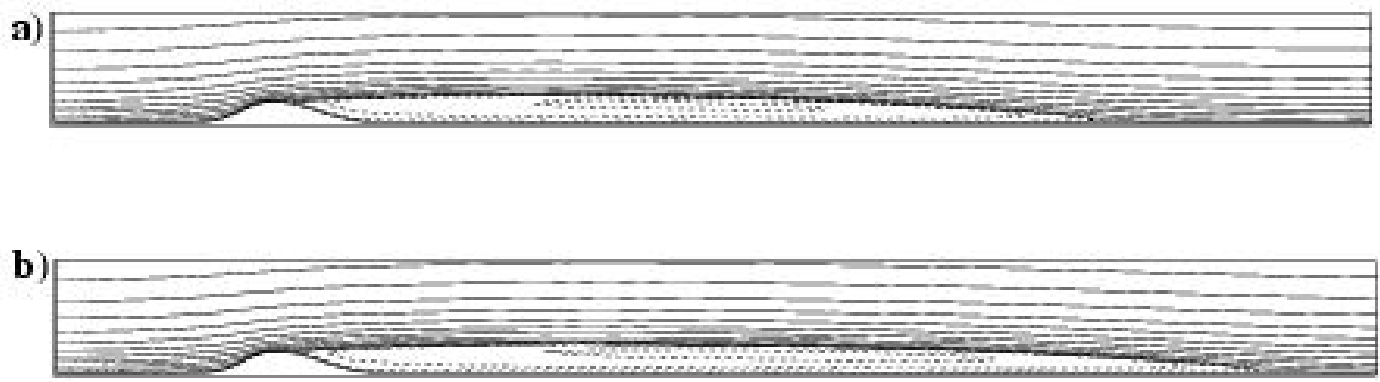

c)

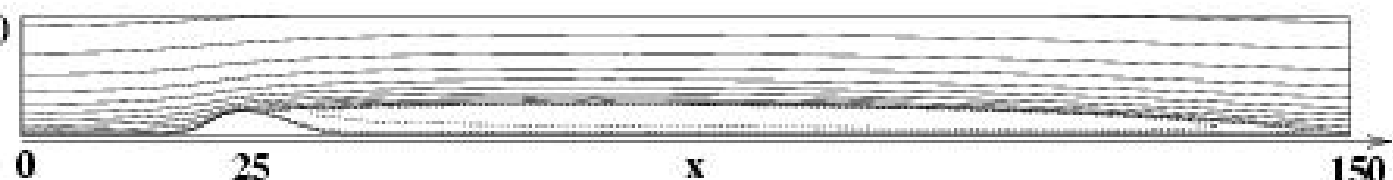

Figure 1. Streamlines of flow states at a) $R e=510, \mathrm{~b}) R e=620$, c) $R e=670$. 
Here, we focus on nonlinear equilibrium states of the stationary Navier-Stokes system for increasing Reynolds number $R e=\delta^{*} U_{\infty}^{*} / \nu$ and hence the flow velocity $\mathbf{u}=(u, v)$ and pressure $p$ are solution of

$$
\mathbf{f}(\mathbf{u}, p, R e)=\left[-(\mathbf{u} \cdot \nabla) \mathbf{u}-\nabla p+(1 / R e) \nabla^{2} \mathbf{u} ; \nabla \cdot \mathbf{u}\right]=\mathbf{0} .
$$

Homogeneous Neumann boundary condition for the flow field $\mathbf{u}$ are imposed at outflow $x=L$, whereas at $y=H$ uniform flow $\mathbf{u}=(1,0)$ is prescribed and the noslip condition applies on the wall boundary. At inflow the Blasius profile $(U(y), 0)$ is imposed. The Chebyshev-Chebyshev discretization is efficient in terms of precision versus grid-size. The problem of spurious pressure modes associated to a Chebyshev discretization in the square is overcome using extra-conditions, by imposing the continuity of the normal derivative of the pressure at the corners and by eliminating the four modes for which the gradient vanishes due to the Chebyshev discretization (cf [12]). A quasi-Newton method, here the Broyden rank-one update procedure (cf [15]), is considered to solve the system (2) for the steady state by adding the Reynolds number as a parameter in an arc-length continuation procedure. The linear systems to be solved during the iterations in the quasi-Newton approach involve the Jacobian matrix

$$
\mathbf{A}(\mathbf{u}, R e)=D_{(\mathbf{u}, p)} \mathbf{f}(\mathbf{u}, R e)
$$

which is evaluated at an initial guess of the flow field and a $Q R$ decomposition is performed. The rank-one update of the decomposition at successive iterations may easily be performed with little extra computational cost (cf. [15]). Once the solution converged to a steady state $\left(\mathbf{u}_{s}, p_{s}\right)$, its stability is computed by considering two-dimensional temporal modes

$$
\mathbf{u}=\hat{\mathbf{u}}(x, y) e^{-i \omega t}, \quad p=\hat{p}(x, y) e^{-i \omega t} .
$$

Taking into account in the Jacobian evaluated at a steady state $\mathbf{u}_{s}$ that the flow perturbation is zero at inflow, the modes are solution of the generalized eigenvalue problem

$$
-i \omega \mathbf{B q}=\mathbf{A}\left(\mathbf{u}_{s}, R e\right) \mathbf{q}
$$

with $\mathbf{q}=(\hat{\mathbf{u}}, \hat{p})$ and $\mathbf{B q}=(\hat{\mathbf{u}}, 0)$. The resulting large eigenvalue problem is solved using large-scale Krylov subspace projections together with the Arnoldi algorithm similar to the approach used in [1]. In all the computations the length of the computational domain is $L=300$ and the height $H=30$. The length of the box proved to be large enough to minimize effects of the box-size on the stability results. The modes are discretized using 250 collocation points in $x$ and 40 collocation points in $y$ and a Krylov subspace with dimension $m=1600$ has been considered.

\section{Basic states and global instability analysis}

The basic states at different Reynolds numbers are depicted in figure 1, the recirculation length increasing with the Reynolds number. In [10] no steady state could be obtained using time integration of the Navier-Stokes system above a Reynolds number of 610 and it has been speculated whether the observed global oscillations 

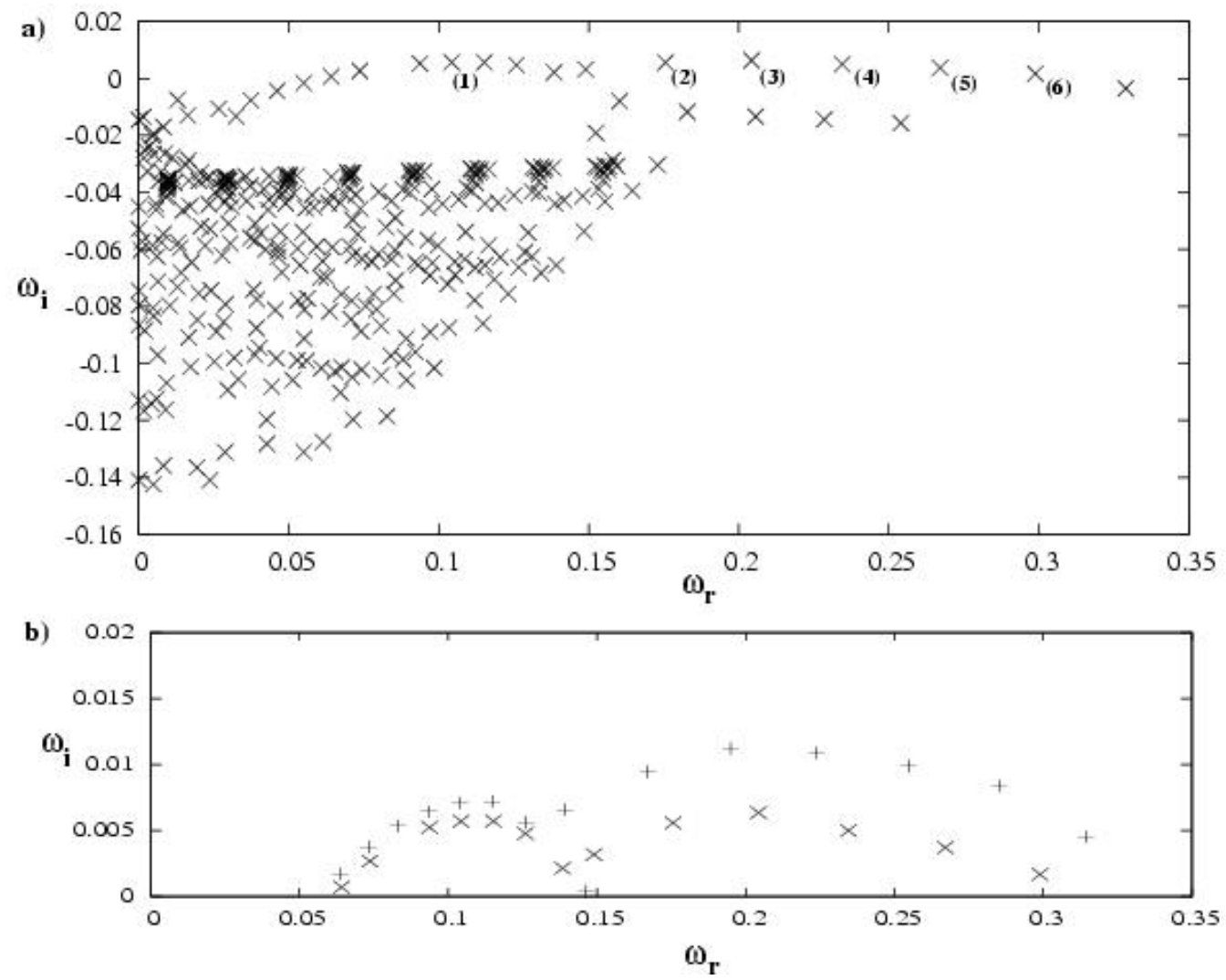

Figure 2. Eigenvalue spectrum at (a) $R e=590$. The modes labelled from (1) - (6) are depicted in figure 3. (b) Unstable part of the spectrum at $R e=590(\times)$ and $R e=620$ $(+)$.

were somehow connected with topological flow changes [16]. With the present nonlinear continuation procedure flow states are computed for higher Reynolds numbers in the unstable range. The results to be discussed hereafter show that the flow at $R e=620$ is indeed unstable. But even at $R e=670$ (cf figure 1c)) there is no evidence of a change in topology.

Once a flow state obtained, its stability is computed with the method outlined in Section 1. Figure 2a) shows the spectrum at $R e=590$ : There are several weakly unstable modes and this Reynolds number hence appears to be slightly above the margin of instability. In figure $2 \mathrm{~b}$ ) only the unstable parts of the spectrum are depicted for comparison at $R e=590,620$, the amplification rates increasing with the Reynolds number. The modes labelled from 1 to 6 are shown in figure 3, figure 3a) depicting the streamlines of the steady state at $R e=590$. One observes that the modes originate approximately at the center of the recirculation bubble. While the mode labelled (1) in figure 2a) at a lower frequency reaches the outflow boundary (cf. figure 3b), the modes (2) to (6) with equally spaced frequencies have the same type of structure, the spatial spreading of the modes decreasing with increasing frequency. The similarity of the eigenmode structure is a typical feature of nonnormal operators and the next section will describe the implication of this. 
a)
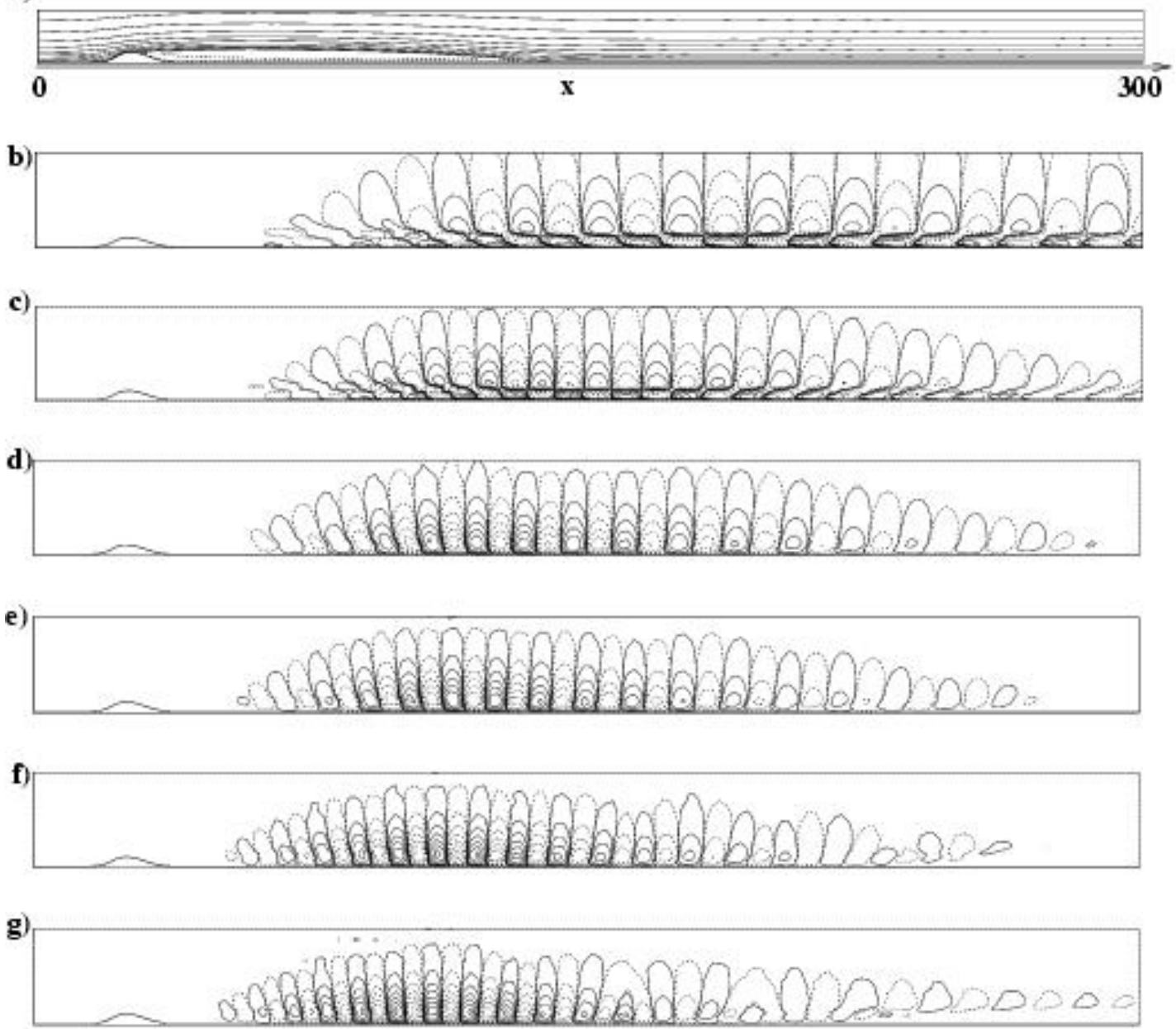

Figure 3. a) Streamlines of basic state at $R e=590$. b)-g) Streamwise velocity components of eigenfunctions corresponding to eigenvalues labelled (1)-(6) in figure 2.

\section{Optimal growth}

When addressing the possibility of growth in a flow system, the notions of optimal initial condition and non-normality of the underlying operators are essential [14]. We are looking for initial disturbances $\mathbf{u}_{0}$ that maximize the energy at time $t$

$$
G(t)=\max _{\mathbf{u}_{0} \neq 0} \frac{\|\mathbf{u}(t)\|_{E}^{2}}{\left\|\mathbf{u}_{0}\right\|_{E}^{2}}
$$

and a convenient form of this expression can be obtained by expanding the solution in terms of the generalized eigenmodes $\mathbf{u}(t)=\sum_{l=1}^{N} \kappa_{l}(t) \hat{\mathbf{u}}_{l}$ with $\hat{\mathbf{u}}_{l}$ solution of (5). Hence the flow dynamics is described by

$$
\frac{d \kappa}{d t}=\Lambda \kappa, \quad \kappa(0)=\kappa_{0},
$$

where $\kappa$ is the vector of expansion coefficients and $\boldsymbol{\Lambda}$ is a diagonal matrix whose elements are given by $\Lambda_{l}=-i \omega_{l}$. The flow perturbation energy in this basis is $\|\mathbf{u}\|_{E}^{2}=\left\|\mathbf{F} \exp (\boldsymbol{\Lambda} t) \kappa_{0}\right\|_{2}^{2}$, where $\mathbf{F}$ is the Cholesky factor of the Hermitian energy 
measure matrix $\mathbf{M}$ with entries $M_{i j}=\int \hat{\mathbf{u}}_{i}^{H} \hat{\mathbf{u}}_{j} d x d y$. Hence, the maximum growth expressed in the basis of eigenmodes reads

$$
G(t)=\left\|\mathbf{F} \exp (\boldsymbol{\Lambda} t) \mathbf{F}^{-1}\right\|_{2}^{2} .
$$

and the largest growth at time $t$ is given by the largest singular value of $\mathbf{F} \exp (\boldsymbol{\Lambda} t) \mathbf{F}^{-1}$ and the optimal initial condition $\kappa_{0}$ is the corresponding right singular vector providing the optimal initial flow condition $\mathbf{u}_{0}$ through the eigenmode expansion.

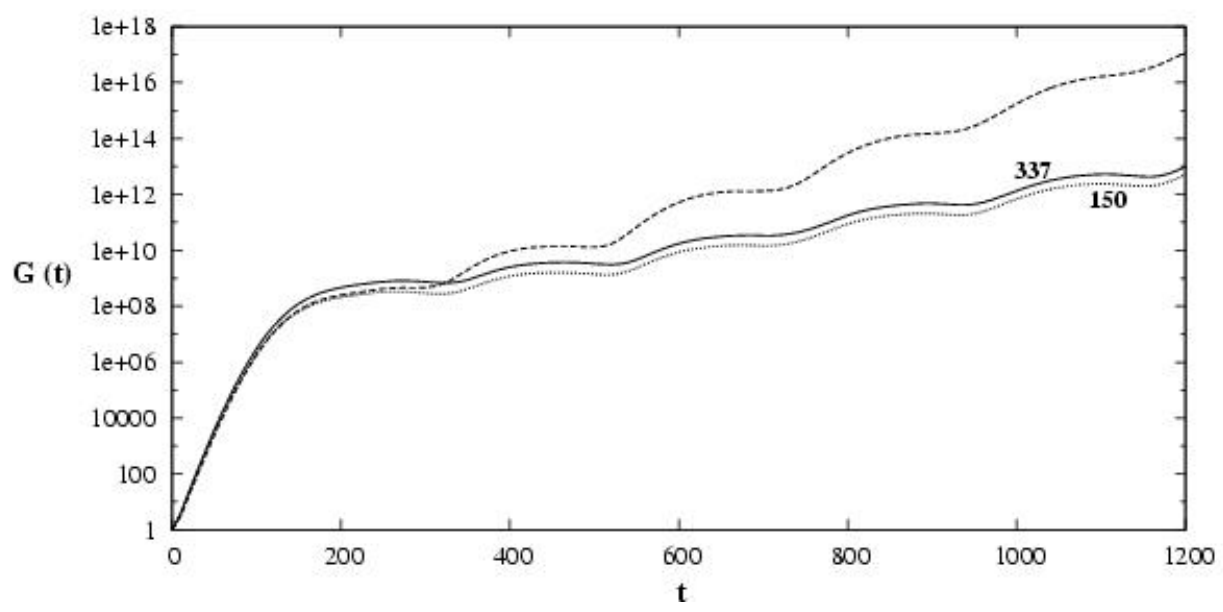

Figure 4. Envelope of maximum energy growth from initial condition, at $R e=590$ with truncation $N=337$ (solid line) and $N=150$ (dotted line). Broken line: envelope at $R e=620$.

The optimal energy gains $G(t)$ are depicted in figure 4, for $R e=590$ and $R e=$ 620. The large Krylov subspace procedure (with $m=1600$ ) gives rise to a set of eigenvalues and its convergence in terms of optimal growth dynamics has been assessed. At $R e=590$ for instance the results with $N=337$ modes and the gain obtained for a much lower truncation at $N=150$ (starting with the most unstable modes) are very close. One observes a fast initial energy growth of a magnitude of almost $10^{9}$ followed by a global cycle with a period close to 200 . This global undulation is accompanied by a weak energy growth due to the amplification rates of the individual modes.

Inspecting figure 2, one observes that the real parts of the unstable eigenvalues in the right half of the spectrum (the modes labelled from (2) to (6)) are distant of about $\delta \approx 0.03$ and the structures of the corresponding eigenmodes are similar. The superposition of these modes as provided by the optimal perturbation analysis gives rise to cancelling leading to the global period of $2 \pi / \delta \approx 200$. Similar non-normal effects have also been described in a recent work on a separated flow in a cavity-like geometry [1].

To illustrate the dynamical behaviour, the time evolution of the perturbation in the eigenmode system is depicted in figure 5 , starting with the optimal initial condition which is mainly located in the vicinity of the rear part of the bump. The perturbation evolves along the plate as a localized wavepacket accompanied with a tremendous increase of energy and at approximately $t=250$ it is leaving the recirculation bubble. At the same time it reappears in the the rear part of the bubble, which is visible at $t=320$. The perturbation than evolves downstream (cf. structure at $t=470$ ) 


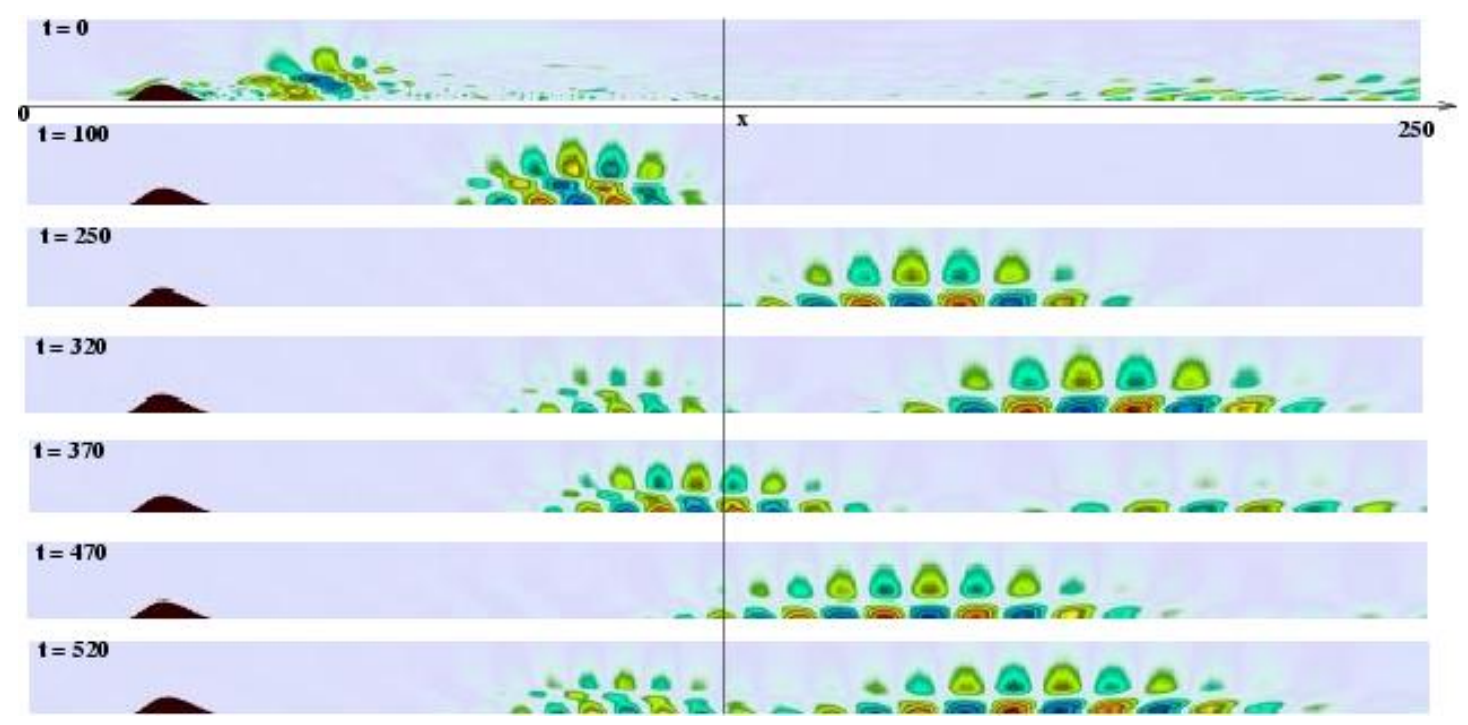

Figure 5. Streamwise velocity component of the perturbation for increasing time from top to the bottom, starting with the optimal initial condition $(R e=590)$. The vertical line shows the location of the reattachment point.

before it reappears again in the bubble (cf. $t=520)$ and the cycle restarts. The modes responsible for the oscillating flow pattern are individually weakly unstable which leads to the overall growth shown in figure 4 for $t>200$.

\section{Direct numerical simulation dynamics}

Focusing on the relation between optimal energy growth and the low-frequency oscillations observed in previous investigations of the separation bubble, the optimal initial condition has been considered in the direct numerical simulation (DNS) procedure of the Navier-Stokes system used in [10].

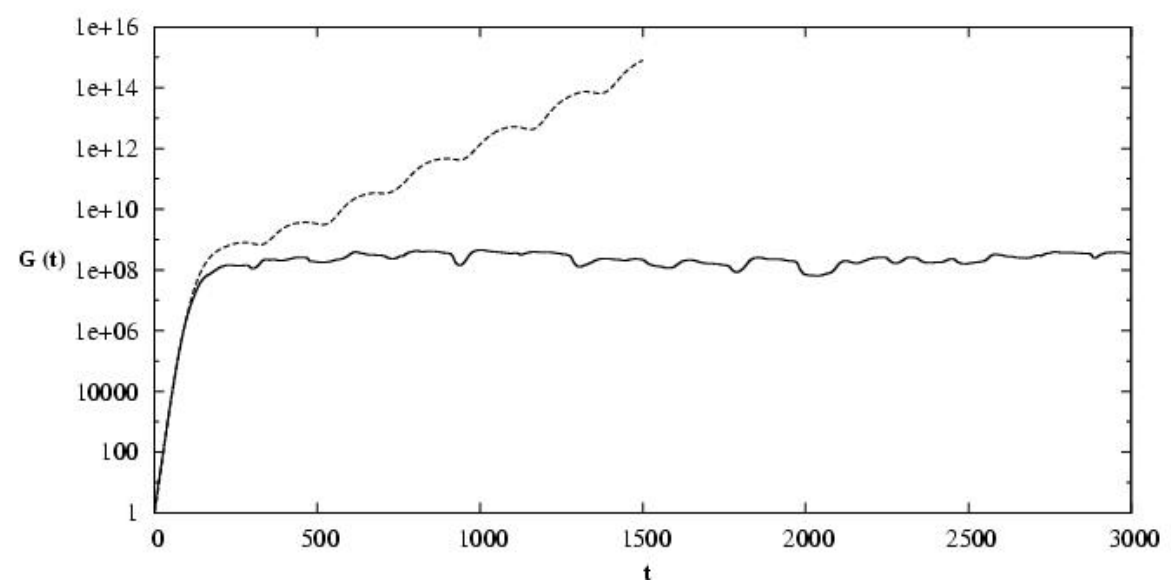

Figure 6. Global energy of $\mathbf{u}-\mathbf{u}_{\mathbf{b}}$ in the time-integrated Navier-Stokes system at $R e=590$, starting with optimal initial condition (solid line). Envelope of the eigenmode system is depicted as the dashed line.

The flow at $R e=590$ is considered, which according to the present analysis is slightly 
supercritical. As shown in [10], at this Reynolds number it was however possible to converge by time marching to a steady state using the DNS procedure. Weak stabilization effects might be attributed to the influence of the outflow boundary of the shorter domain considered in the DNS (with $L=200$ ), where a classical convection condition is applied. The streamwise direction is discretized using fourthorder finite differences with 1024 equidistant grid points. In $y$ the region up to $H=$ 80 is covered (with 97 Chebyshev-collocation points) for the solution to be uniform in the upstream region, a simple linear coordinate transform being employed. The gain in energy being expected to be close to $10^{9}$, the optimal initial condition has been affected with a small maximum amplitude of $510^{-5}$ (however above the residual noise of order of $10^{-6}$ in the time-marching towards the steady basic state $\mathbf{u}_{b}$ ). The energy of the perturbation $\mathbf{u}^{\prime}=\mathbf{u}-\mathbf{u}_{b}$ has been integrated in the whole domain and the gain is depicted in figure 6 . Up to $t=200$ the energy growth follows closely the curve provided by the eigenmode system before nonlinear saturation occurs. The integration has been pursued up to $t=3000$ and the flow is seen to remain in a nonlinear state. When entering the nonlinear saturation, the oscillations visible in the global energy curve are somehow reminiscent of the global oscillations in the envelope curve provided by the modes. The amplitude of the nonlinear flow

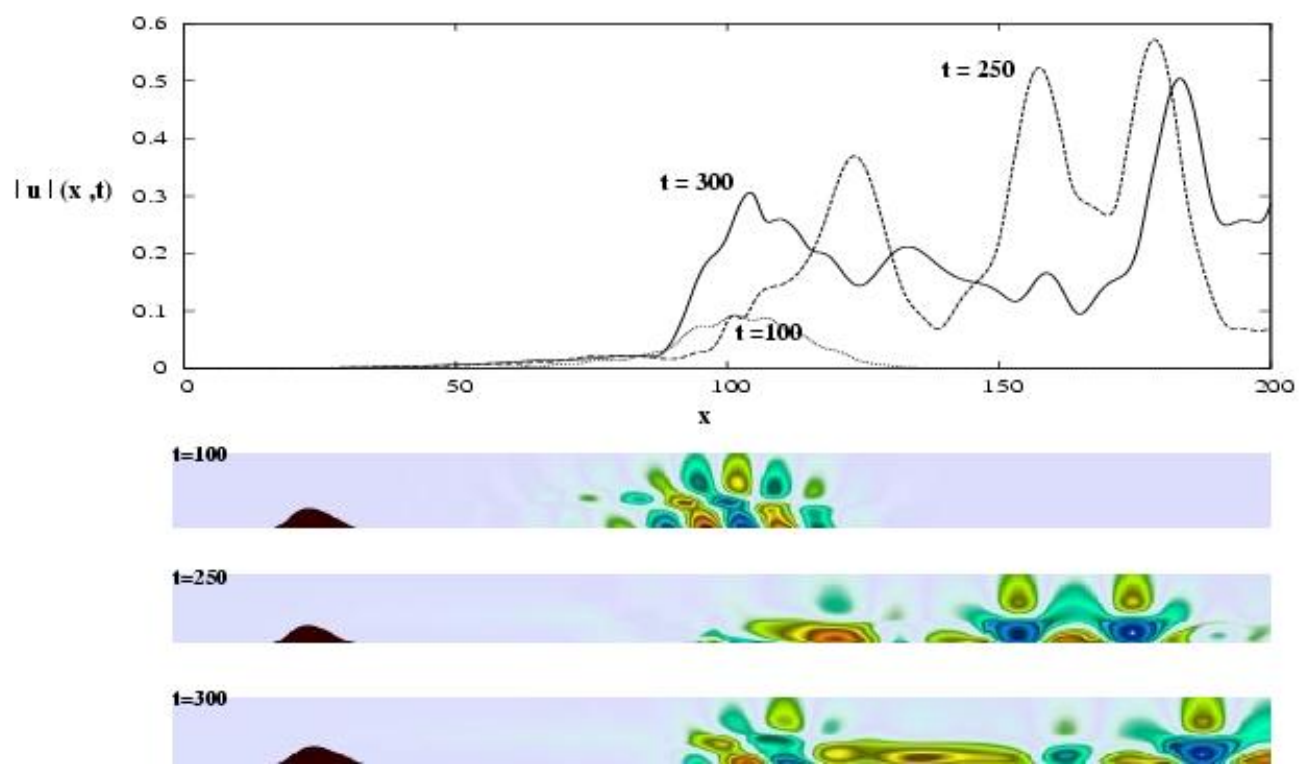

Figure 7. a) Amplitude $\int\left\|\mathbf{u}^{\prime}\right\| d y$ of perturbation flow field in the DNS, as function of $x$, at various times. b)-d) Instantaneous streamwise velocity components of the perturbation flow field.

perturbation, by simply subtracting the base flow, along the plate at increasing time has been computed and the results are shown in figure 7. At $t=100$ the perturbation is similar to that depicted in figure 5 for the dynamical system formed with the global modes. At $t=300$ a regeneration behaviour of the perturbation is visible upstream the previous wavepacket at $t=250$. The perturbation is highly nonlinear and is hence far from being a superposition of eigenmodes.

In [10] low-frequency oscillations of the bubble have been reported at the supercritical Reynolds number $R e=650$. In the present analysis similar fluctuations are observed as a consequence of the initial optimal perturbation at $R e=590$. Figure 


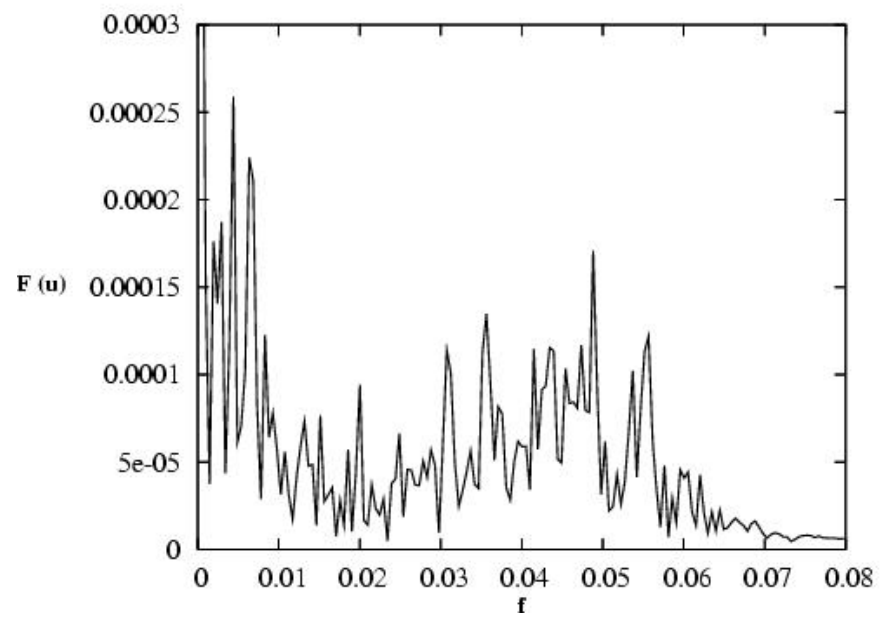

Figure 8. Power spectrum in time $500 \leq t \leq 3000$ of streamwise velocity component at $x=40, y=1$.

8 depicts the amplitude of the Fourier transform for the streamwise velocity profile at $x=60, y=1$, that is at a position which corresponds approximately to the center of the steady bubble. The low frequency $f=\delta /(2 \pi) \approx 0.005$ corresponds to the non-normal 'beating' frequency in the eigenmodes system and indeed the spectrum exhibits peaks in this frequency range, the nonlinear flow being highly aperiodic. Similar to the results reported in [10] a second dominant range of higher frequency is visible. Figure 9 shows an instantaneous vorticity field illustrating the vortex-shedding behaviour resulting from the two-dimensional global oscillations.

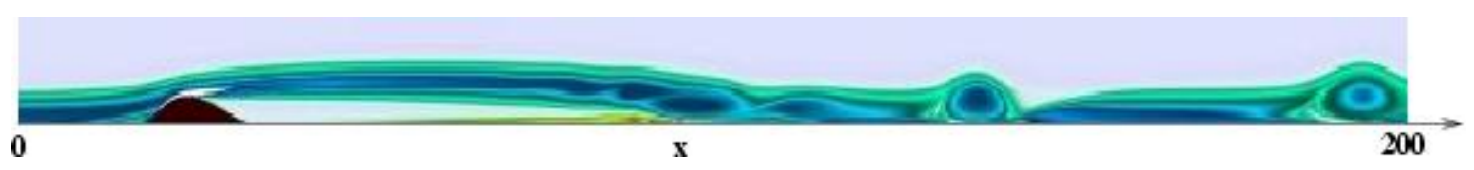

Figure 9. Instantaneous vorticity at $t=1500$.

\section{Conclusion}

In the aim of shedding new light on the phenomenon of global 'flapping' in separated wall-bounded flows, the two-dimensional flow over a bump has been considered, by computing nonlinear states of the stationary Navier-Stokes system. When increasing the Reynolds number, no topological changes in the flow structure are detected.

The separation bubble becomes unstable with respect to two-dimensional temporal modes starting at the center of the bubble and extending more or less downstream the reattachment point. The corresponding eigenvalues cross the axis of marginal instability almost simultaneously and they exhibit equally spaced frequencies.

By performing an optimal initial disturbance analysis, a periodic regeneration mechanism of the perturbation at the rear of the bubble, resulting from non-normal cancelling of eigenmode structures, has been detected. The time-integration of the Navier-Stokes system starting with the optimal initial condition leads to nonlinear aperiodic flow with however the reminiscence of a global low-frequency oscillation of 
the bubble. This gives some strength to the conjecture, that non-normal interaction of global modes is at the origin of the 'flapping' behaviour.

\section{Acknowledgements}

Parts of the computations have been performed on the NEC-SX8 of the IDRIS, France. The authors would like to thank Matthieu Marquillie for providing the DNS code.

\section{References}

[1] E. Åkervik, J. Hopffner, U. Ehrenstein, and D. Henningson. Optimal growth, model reduction and control in a separated boundary-layer flow using global eigenmodes. J. Fluid Mech., 2007. in press.

[2] D. Barkley, M. Gomes, and D. Henderson. Three-dimensional instability in flow over a backward-facing step. J. Fluid Mech., 473:167-189, 2002.

[3] J.-F. Beaudoin, O. Cadot, J.-L. Aider, and J. Wesfreid. Three-dimensional stationary flow over a backward-facing step. Eur. J. Mech. B/Fluids, 23:147-155, 2004.

[4] N.J. Cherry, R. Hiller, and M.P. Latour. Unsteady measurements in a separating and reattaching flow. J. Fluid Mech., 144:13- 46, 1984.

[5] U. Dallmann, Th. Herberg, H. Gebing, W.-H. Su, and H.-Q. Zhang. Flow field diagnostics: topological flow changes and spatio-temporal flow structure. AIAA Paper 95-0791, 1995.

[6] A.V. Dovgal, V.V. Kozlov, and A. Michalke. Laminar boundary layer separation: instability and associated phenomena. Prog. Aerospace Sci., 30:61- 94, 1994.

[7] F. Gallaire, M. Marquillie, and U. Ehrenstein. Three-dimensional transverse instabilities in detached boundary-layers. J. Fluid Mech., 571:221-233, 2007.

[8] C. P. Häggmark, A.A. Bakchinov, and P.H. Alfredsson. Experiments on a two-dimensional laminar separation bubble. Phi. Trans. R. Soc. Lond. A., 358:3193-3205, 2000.

[9] D.A. Hammond and L.G. Redekopp. Local and global instability properties of separation bubbles. Eur. J. Mech. B/Fluids, 17:145-164, 1998.

[10] M. Marquillie and U. Ehrenstein. On the onset of nonlinear oscillations in a separating boundary-layer flow. J. Fluid Mech., 490:169- 188, 2003.

[11] L.L Pauley, P. Moin, and W.C. Reynolds. The structure of two-dimensional separation. J. Fluid Mech., 220:397- 411, 1990.

[12] R. Peyret. Spectral Methods for Incompressible Flows. Springer, 2002.

[13] U. Rist and U. Maucher. Investigations of time-growing instabilities in laminar separation bubbles. Eur. J. Mech. B/Fluids, 21:495- 509, 2002.

[14] P. J. Schmid and D.S. Henningson. Stability and Transition in Shear Flows. Springer, 2001.

[15] J. Stoer and R. Bulirsch. Introduction to Numerical Analysis. Springer, 1992.

[16] V. Theofilis, S. Hein, and U. Dallmann. On the origins of unsteadiness and threedimensionality in a laminar separation bubble. Phil. Trans. R. Soc. Lond. A, 358:3229$3246,2000$. 